\title{
Nanostructured core optical fibres (Conference Presentation) (Withdrawal Notice)
}

Ryszard Buczynski, Alicja Anuszkiewicz, Marcin Franczyk, Adam Filipkowski, Tomasz Osuch, et al.

Ryszard Buczynski, Alicja Anuszkiewicz, Marcin Franczyk, Adam Filipkowski, Tomasz Osuch, Dariusz Pysz, Tomasz Stefaniuk, Grzegorz Stepniewski, Damian Michalik, Hue Thi Nguyen, Mariusz Klimczak, Rafal Kasztelanic, "Nanostructured core optical fibres (Conference Presentation) (Withdrawal Notice)," Proc. SPIE 11029, Micro-structured and Specialty Optical Fibres VI, 1102906 (19 May 2019); doi: 10.1117/12.2524881

SPE. Event: SPIE Optics + Optoelectronics, 2019, Prague, Czech Republic 


\title{
Nanostructured core optical fibres (Conference Presentation) (Withdrawal Notice)
}

\author{
Ryszard Buczynski, ${ }^{1,2}$ Alicja Anuszkiewicz, ${ }^{1}$ Marcin Franczyk, ${ }^{1}$ Adam Filipkowski, ${ }^{1}$ Tomasz Osuch, ${ }^{3}$ \\ Dariusz Pysz, ${ }^{1}$ Tomasz Stefaniuk, 2,1 Grzegorz Stepniewski, ${ }^{1}$ Damian Michalik, 1,2 Hue Thi Nguyen,, ${ }^{2}$ \\ Mariusz Klimczak, ${ }^{1}$ Rafal Kasztelanic ${ }^{1,2}$ \\ IInstitute of Electronic Materials Technology (Poland) \\ 2Univ. of Warsaw (Poland) \\ 3Warsaw Univ. of Technology (Poland) \\ Proceedings Volume 11029, Micro-structured and Specialty Optical Fibres VI; 1102906 (2019) \\ https://doi.org/10.1117/12.2524881
}

Event: SPIE Optics + Optoelectronics, 2019, Prague, Czech Republic

Online Publication Date: 14 May 2019

Withdrawn from Publication: 19 May 2019

Publisher's Note: This conference presentation, originally published on 14 May 2019, was withdrawn per author request. 